\title{
Uncertainty Modeling in Human Health Risk Assessment using Fuzzy Set
}

\author{
Tabendra Nath Das \\ Dept. of Mathematics \\ Dhakuakhana College \\ Dhakuakhana-787055
}

\author{
Palash Dutta \\ Dept. of Mathematics \\ Dibrugarh University \\ Dibrugarh-786004
}

\begin{abstract}
Risk assessment is an important and popular aid in decision making process. Risk assessment is generally performed using models and model is a function of some parameters. Sometimes model parameters are tainted with uncertainty due to lack of knowledge, imprecision, vagueness, small sample sizes etc. To represent this type of uncertainty generally triangular fuzzy numbers or trapezoidal fuzzy numbers are used. In this paper, we use Gaussian and Cauchy fuzzy numbers to represent uncertainty. Fusion of Gaussian and Cauchy fuzzy number is discussed and then human health risk assessment is carried out in this setting.
\end{abstract}

\section{KEY WORDS}

Human Health Risk Assessment, Uncertainty, Fuzzy Set, Gaussian \& Cauchy Fuzzy Numbers.

\section{INTRODUCTION}

Risk assessment is an important and integral part of decision making process. Risk assessment is generally performed using models and model is a function of parameters. Usually model parameters are tainted with uncertainty. It may be either aleatory or epistemic uncertainty. Aleatory uncertainty arises due to inherent variability, natural stochasticity, environmental or structural variation across space or through time, manufacturing or genetic heterogeneity among components or individuals, and Varity of others sources of randomness. On the other hand epistemic uncertainty arises due to the insufficient knowledge about the world, which includes small sample sizes, detection limits, imperfections in scientific understanding etc. More often environmental data tend to be vague and imprecise so uncertainty is always involves in the study of risk assessment

If the representation of the input parameters of the risk model is in probabilistic sense, the output risk distribution may reflect probabilistic information. However, resalable and sufficient data is required to estimate and characterized the probability distribution of the input variables. If uncertainty does not arise due to randomness, or if the information are partial, not fully reliable, receive of information from more than one sources or inherent imprecision, then probability theory is inappropriate to represent such kind of uncertainty. To overcome this limitation of probability theory Zadeh (1965) introduced fuzzy set theory which can be used to incorporate epistemic uncertainty.

In this study, it is considered that model parameters are tainted with uncertainty due to lack of knowledge, imprecision, vagueness, small sample sizes, measurement error etc. In general, Triangular or Trapezoidal shape fuzzy membership functions have been widely studied in literature to represent this type of uncertainty. However, in practice, there are certain applications where to represent uncertainty, besides Triangular or Trapezoidal shape fuzzy numbers some other types of fuzzy numbers come into picture viz., Gaussian fuzzy number, lognormal shaped fuzzy number, Cauchy shaped fuzzy number etc. In [4] authors discussed Gaussian and triangular fuzzy numbers and performed arithmetic operations between Gaussian fuzzy number and triangular fuzzy number and applied it in risk assessment models. Also in [5] authors fused Cauchy and Triangular fuzzy numbers and applied the fusion technique in health risk assessment model.

In this paper, Gaussian and Cauchy fuzzy numbers are used to represent epistemic type uncertainty. An attempt has been made to combine Gaussian fuzzy number with Cauchy fuzzy number and then human health risk assessment is carried out using proposed combined technique.

\section{PRELIMINARIES}

To estimate the effects of environmental pollution on human, risk assessment is performed. However, environmental data tends to be vague and imprecise, so uncertainty is associated with any study related with risk assessment. Fuzzy set theory is a tool which is used to characterize imprecisely defined variables, as well as to define relationships between variables based on expert knowledge and use them to compute results. In this section, [2, 3] some necessary backgrounds and notions of fuzzy set theory are reviewed.

Definition 2.1.: Let $X$ be a universal set. Then the fuzzy subset $A$ of $X$ is defined by its membership function

$$
\mu_{A}: X \rightarrow[0,1]
$$

which assign a real number $\mu_{A}(x)$ in the interval $[0,1]$, to each element $x \in X$, where the value of $\mu_{A}(x)$ at $x$ shows the grade of membership of $x$ in $A$.

Definition 2.2.: Given a fuzzy set $A$ in $X$ and any real number $\boldsymbol{\alpha} \in[0,1]$, the $\boldsymbol{\alpha}$-cut or $\boldsymbol{\alpha}$-level or cut worthy set of $A$, denoted by ${ }^{\alpha} A$ is the crisp set

$$
{ }^{a} A=\left\{x \in X: \mu_{A}(x) \geq \boldsymbol{\alpha}\right\}
$$

The strong $a$ cut, denoted by ${ }^{\alpha+} A$ is the crisp set

$$
{ }^{a+} A=\left\{x \in X: \mu_{A}(x)>\boldsymbol{\alpha}\right\}
$$

For example, let A be a fuzzy set whose membership function is given as

$$
\mu_{A}(x)=\left\{\begin{array}{l}
\frac{x-a}{b-a}, a \leq x \leq b \\
\frac{c-x}{c-b}, b \leq x \leq c
\end{array}\right.
$$


To find the $\alpha$-cut of A, we first set $\alpha \in[0,1]$ to both left and right reference functions of $\mathrm{A}$.

$$
\text { That is, } \alpha=\frac{x-a}{b-a} \text { and } \alpha=\frac{c-x}{c-b} \text {. }
$$

Expressing $\mathrm{x}$ in terms of $\alpha$ we have $x=(b-a) \alpha+a$ and $x=c-(c-b) \alpha$, which gives the $\alpha$-cut of $\mathrm{A}$ is

$$
\alpha_{A=[(b-a) \alpha+a, c-(c-b) \alpha]}
$$

Definition 2.3.: The support of a fuzzy set $A$ defined on $X$ is a crisp set defined as

$$
\operatorname{Supp}(A)=\left\{x \in X: \mu_{A}(x)>0\right\}
$$

Definition 2.4.: The height of a fuzzy set $A$, denoted by $h(A)$ is the largest membership grade obtain by any element in the set.

$$
h(A)=\sup _{x \in X} \mu_{A}(x)
$$

Definition 2.5: A fuzzy number is a convex normalized fuzzy set of the real line $R$ whose membership function is piecewise continuous.

\subsection{Type of fuzzy numbers}

\subsubsection{Triangular Fuzzy Number}

A triangular fuzzy number A can be defined as a triplet $[a, b$, $c]$. Its membership function is defined as:

$$
\mu_{A}(x)= \begin{cases}\frac{x-a}{b-a} & , a \leq x \leq b \\ \frac{c-x}{c-b} & , b \leq x \leq c\end{cases}
$$

\subsubsection{Trapezoidal Fuzzy Number}

A trapezoidal fuzzy number $A$ can be expressed as $[a, b, c, d]$ and its membership function is defined as:

$$
\mu_{A}(x)= \begin{cases}\frac{x-a}{b-a}, & a \leq x<b \\ 1, & , b \leq x \leq c \\ \frac{d-x}{d-c}, & c<x \leq d\end{cases}
$$

\subsubsection{Gaussian Fuzzy number}

The membership function of a Gaussian Fuzzy number is $[4,6]:$

$$
\mu_{A}(x)=\exp \left(\frac{-(x-\mu)^{2}}{2 \sigma^{2}}\right)
$$

where $\mu$ represents the MFs centre and $\sigma$ determines the MFs width.

\subsubsection{Cauchy Fuzzy number:}

The membership function of a Cauchy Fuzzy number is $[1,6]$ :

$$
\mu_{A}(x)=\frac{1}{1+\left(\frac{x-p}{q}\right)^{2}}
$$

Where $\mathrm{p}$ represents the MFs centre and q determines the MFs width.

\section{AN APPROACH TO COMBINE GAUSSIAN AND CAUCHY FUZZY NUMBER}

In this section, arithmetic operations between Gaussian and Cauchy fuzzy numbers have been performed using $\alpha$-cut technique.

The general form of the $\alpha$-cut of Gaussian fuzzy number is

$$
{ }^{\alpha} A_{1}=[\mu-\sigma \sqrt{-2 \ln \alpha}, \mu+\sigma \sqrt{-2 \ln \alpha}]
$$

Also the general form of the $\alpha$-cut of Cauchy fuzzy number is

$$
\alpha_{A_{2}}=\left[p-q \sqrt{\frac{1-\alpha}{\alpha}}, p+q \sqrt{\frac{1-\alpha}{\alpha}}\right]
$$

Here, the condition of convexity has been relaxed to combine Cauchy fuzzy number and triangular fuzzy number using $\alpha$ cut by considering that $\alpha$ corresponds to the $\alpha$-cut defined in the interval $[0.01,1]$. It will not affect the uncertainty involved in the fuzzy number.

\subsection{Addition}

$$
{ }^{\alpha} A_{1}+{ }^{\alpha} A_{2}=\left[\mu-\sigma \sqrt{-2 \ln \alpha}+p-q \sqrt{\frac{1-\alpha}{\alpha}}, \mu+\sigma \sqrt{-2 \ln \alpha}+p+q \sqrt{\frac{1-\alpha}{\alpha}}\right]
$$

\subsection{Subtraction}

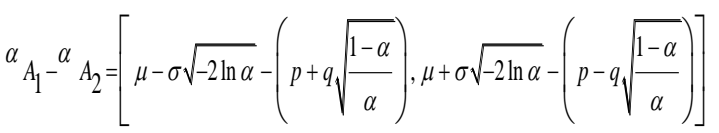

\subsection{Multiplication}

$$
\alpha_{A_{1}{ }^{\alpha} A_{2}}=\left[(\mu-\sigma \sqrt{-2 \ln \alpha})\left(p-q \sqrt{\frac{1-\alpha}{\alpha}}\right),(\mu+\sigma \sqrt{-2 \ln \alpha})\left(p+q \sqrt{\frac{1-\alpha}{\alpha}}\right)\right]
$$

\subsection{Division}

$$
\frac{{ }_{A_{1}}}{\alpha_{A_{2}}}=\left[\frac{(\mu-\sigma \sqrt{-2 \ln \alpha})}{\left(p+q \sqrt{\frac{1-\alpha}{\alpha}}\right)}, \frac{(\mu+\sigma \sqrt{-2 \ln \alpha})}{\left(p-q \sqrt{\frac{1-\alpha}{\alpha}}\right)}\right]
$$

\section{HYPOTHETICAL CASE STUDY}

To demonstrate and make use of the Gaussian fuzzy number and Cauchy fuzzy numbers and their combination a hypothetical case study for health risk assessment is presented here.

Suppose the exposure of a population to chlorinated organic solvent $(1,1,2$-trichloroethene), via the consumption of contaminated drinking water. The general form of a risk assessment model for a lifetime daily dose of exposure as provided by EPA, 2001 is follows [7]:

$$
D=\frac{C D I \times I R \times E F \times E D}{B W \times A T}
$$


Where, $D$ is the dose of exposure $(\mathrm{mg} / \mathrm{Kg}$.day), $I R$ is the intake of water $(1 / d), C D I$ is the concentration of $1,1,2$ trichloroethene in the water $(\mathrm{mg} / \mathrm{l}), E F$ is the exposure frequency (d/yr), $E D$ exposure duration (yr), $B W$ is the body weight $(\mathrm{kg}), A T$ is the average time (d).

The excess risk is express as:

$$
E R=D \times U E R
$$

$U E R$ is the unit excess risk: a probability of excess cancer per unit daily dose of exposure.

In this study, the parameter intake rate of water $(I R)$ is considered as triangular fuzzy number and exposure duration $(E D)$ is considered to be Cauchy fuzzy number while other parameters are taken to be constant. The parameters values used in the risk assessment are given in table1.

Table1: Parameter values used in the risk assessment.

\begin{tabular}{|c|c|c|}
\hline Variable & Representation & Value \\
\hline$C D I$ & Deterministic & 0.2 \\
\hline$I R$ & Possibilistic & *GFN $(1.5,0.195)$ \\
\hline$E F$ & Deterministic & 250 \\
\hline$E D$ & Possibilistic & $\mathrm{CFN}(30,2)$ \\
\hline$B W$ & Deterministic & 70 \\
\hline$\overline{A T}$ & Deterministic & 70 \\
\hline UER & Deterministic & 0.024 \\
\hline
\end{tabular}

As the two input parameters of the health risk assessment model are fuzzy numbers so the resulting risk will be obtained in the form of fuzzy number and its graphical representation is given in the figure 1 .

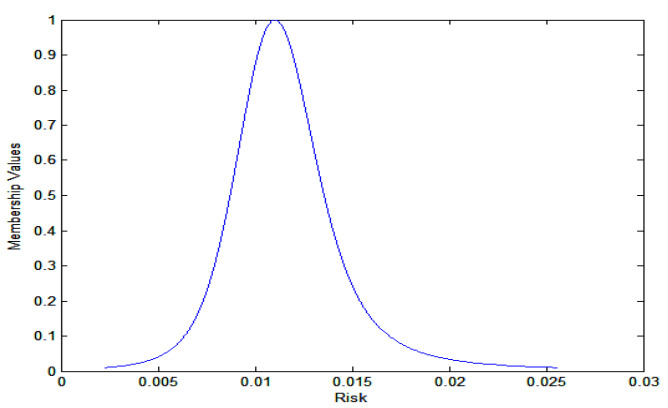

Figure1: Human helth risk assessment
Here, the core or modal value of the resulting health risk is 0.01102 while range of risk value is [0.002246, 0.02556].

\section{CONCLUSION}

Risk assessment is a popular and important tool in decision making process. Risk assessment is generally performed using models and model is a function of some parameters which are usually affected by uncertainty. Since it is considered that the parameters are tainted with epistemic uncertainty, the same is propagated to the output through the model. In general, Triangular or Trapezoidal shape fuzzy membership functions have been widely studied in literature to represent uncertainty. However, in practice, there are certain applications where to represent uncertainty besides Triangular or Trapezoidal shape fuzzy numbers some other types of fuzzy numbers come into picture viz., Gaussian fuzzy number, lognormal shaped fuzzy number, Cauchy fuzzy number etc. In this paper, Gaussian and Cauchy fuzzy numbers have been studied and an effort has been made to fuse them. Finally, proposed fusion technique has been applied in health risk assessment model to demonstrate and check its validity. In the model one parameter is considered as Gaussian fuzzy number and another one is considered as Cauchy fuzzy number, and other parameters are taken to be constant. Therefore, the resulting risk is also obtained in the form of a fuzzy number. 0.01102 is the core of the output fuzzy risk while the range is [0.002246, $0.02556]$

\section{REFERENCES}

[1] Ashraf M. A., Suzan A., Donald C. W. (2007) Gaussian Versus Cauchy Membership Functions in Fuzzy PSO, Proceedings of International Joint Conference on Neural Networks, Orlando, Florida, USA.

[2] Bojadziev G. and Bojadziev, M. (1995), Fuzzy set, Fuzzy logic, application, (Singapore: world Scientific.)

[3] Dutta P., Boruah H. and Ali T. (2011), Fuzzy arithmetic with and without using $\alpha$-cut method: a comparative study", International Journal of Latest Trends in Computing, 2 (1): 99-108.

[4] Dutta P., Ali T. (2012), Uncertainty Modeling in Risk Analysis: A Fuzzy Set Approach, International Journal of Computer Applications, 43 (17): 35-39.

[5] Dutta P., (2013), Fusion of Cauchy and Triangular Fuzzy Numbers: Application in Human Health Risk Assessment, Elixir Applied Mathematics, 63: 1816618171.

[6] Jang J.S.R., Sun C.T., Mizutani E. (1996) Neuro-Fuzzy and Soft Computing, Prentice-Hall.

[7] US EPA. (2001). Risk Assessment Guidance for Superfund, Volume I: Human Health Evaluation Manual (Part E, Supplemental Guidance for Dermal Risk Assessment). Office of Emergency and Remedial Response, EPA/540/R/99/005, Interim, Review Draft. United States Environmental Protection Agency. 